
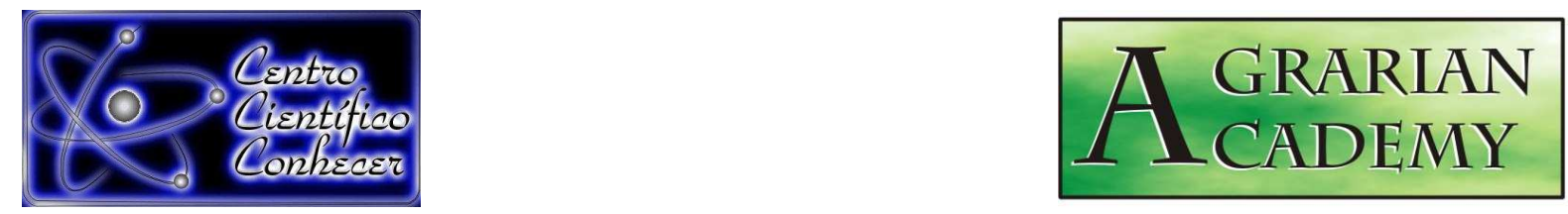

\title{
ADAPTABILIDADE DE BEZERROS MESTIÇOS (HOLANDÊS X GIR) ÀS CONDIÇÕES AMBIENTAIS DO SEMIÁRIDO PIAUIENSE
}

\author{
Jardênio Assis dos Santos ${ }^{1}$, Luiz Alexandre Silva Camapum ${ }^{1}$, Tairon Pannunzio Dias \\ e Silva ${ }^{2^{*}}$, Alécio Matos Pereira ${ }^{3}$ \\ ${ }^{1}$ Graduando em Medicina Veterinária - CPCE/UFPI, Bom Jesus, Piauí, Brasil \\ ${ }^{2}$ Mestre em Zootecnia - CPCE/UFPI, Doutorando em Ciências, Centro de Energia \\ Nuclear na Agricultura (CENA), Universidade de São Paulo (USP), Piracicaba, São \\ Paulo, Brasil (tairon.mvet@gmail.com) \\ ${ }^{3}$ Professor da Universidade Federal do Piauí (UFPI), Campus Professora Cinobelina \\ Elvas (CPCE), Bom Jesus, Piauí, Brasil
}

Recebido em: 03/01/2014 - Aprovado em: 04/04/2014 - Publicado em: 12/04/2014

\begin{abstract}
RESUMO
Objetivou-se avaliar os parâmetros fisiológicos de bezerros mestiços (Holandês $x$ Gir) no semiárido piauiense. Foram utilizados 12 bezerros em Delineamento Inteiramente Casualizado e esquema fatorial 2x2 (2 sexos, machos e fêmeas e 2 turnos, manhã e tarde) com oito repetições. Os parâmetros fisiológicos, frequência cardíaca (FC), frequência respiratória ( $F R$ ) e temperatura retal (TR), foram aferidos nos períodos da manhã e da tarde. Foram constatadas diferenças $(P<0,05)$ entre os turnos para todas as variáveis bioclimáticas. Avaliando a TA (temperatura ambiente), esta caracteriza-se fora da zona de conforto térmico para bovinos indianos. Percebese que o ITGU apresentou valores maiores durante a tarde, caracterizando o ambiente com situação de emergência. Analisando os diferentes sexos dentro de turno verifica-se que os animais fêmeas apresentaram uma maior utilização da FR durante o turno da tarde, possivelmente na tentativa de dissipação de calor corporal. Com relação a FC percebe-se uma semelhança entre sexo e turno, não diferindo $(P>0,05)$. Verificou-se efeito de turno sobre a TR de machos e fêmeas, provavelmente devido ao aumento da carga térmica adicional recebida da radiação solar. Inferindo-se que os valores de TR das bezerras, apresentou superioridade e diferiu $(P<0,05)$ dos bezerros no turno da tarde. Bezerras são mais susceptíveis às condições ambientais estressantes da região do vale do Gurguéia, utilizando em maior intensidade os mecanismos termorreguladores no turno da tarde.
\end{abstract}

PALAVRAS-CHAVE: bem-estar, bovinos, estresse, parâmetros fisiológicos, termorregulação

\section{ADAPTABILITY OF CROSSBRED CALVES (HOLSTEIN X GIR) ENVIRONMENTAL CONDITIONS OF PIAUÍ SEMIARID}

\section{ABSTRACT}

This study aimed to evaluate the physiological parameters of crossbred calves (Holstein $x$ Gir) in the semiarid region of Piauí. 12 calves were used in a completely randomized design in a factorial scheme $2 \times 2$ (two sexes, male and female, and 2 shifts, morning and afternoon) with eight replicates. Physiological parameters, heart 
rate $(H R)$, respiratory rate $(R R)$ and rectal temperature $(R T)$ were measured in the morning and afternoon. Differences were observed $(P<0.05)$ between shifts for all bioclimatic variables. Assessing the $T$, this is characterized outside the zone of thermal comfort for Indian cattle. It is noticed that BGT had higher values in the afternoon, characterizing the environment with the emergency. Analyzing the different sexes in turn verifies that the female animals showed greater use of FR during the afternoon, possibly in an attempt to dissipate body heat. Regarding FC perceive a similarity between the shift between sex and did not differ $(P>0.05)$. There was no effect on TR shift of males and females, probably due to the increased thermal load additional incoming solar radiation. Inferring that the TR values in calves showed superiority and differed $(P<0.05)$ of calves in the afternoon. Calves are more susceptible to environmental conditions stressful Valley region Gurguéia using higher intensity in the thermoregulatory mechanisms in the afternoon.

KEYWORDS: cattle, physiological parameters, welfare, stress, thermoregulation

\section{INTRODUÇÃO}

O estresse calórico aumenta à medida que a umidade relativa e a temperatura ambiente ultrapassam a zona de conforto térmico, dificultando assim a dissipação de calor que, por sua vez, aumenta a temperatura corporal com efeito negativo sobre o desempenho produtivo dos bovinos (SILVA et al., 2012).

Avaliar se o animal encontra-se em ambiente ótimo ou estressante, é de fundamental importância para a eficiência fisiológica e econômica da exploração. Nesse sentido, torna-se indispensável avaliar o desempenho produtivo, fisiológico e comportamental do animal em um determinado ambiente. A maioria dos estudos tem se desenvolvido em condições térmicas controladas, através do uso de câmaras climáticas, as quais não refletem as flutuações normalmente verificadas ao longo do dia nas condições naturais. É de suma importância estudos relativos ao comportamento de bovinos leiteiros na ausência de sistemas de climatização permitindo assim, conhecer de que forma a alteração do comportamento pode afetar atividades como pastejo, ruminação e, consequentemente, 0 crescimento $e$ desempenho produtivo (KENDALL et al., 2006).

Os conhecimentos gerados a partir do comportamento animal são fundamentalmente importantes no acompanhamento e adequação do sistema de produção como um todo, incluindo atividades individuais e seus ambientes sociais e físicos, sendo possível uma melhor compreensão das causas que norteiam as ações dos animais (STRICKLIN \& KAUTZ-SCANAVY, 1984). Em alguns casos, mudanças nos padrões do comportamento são as únicas indicações visíveis de que o estresse está presente (PERISSINOTO et al., 2009).

De forma geral os animais procuram ambientes mais confortáveis a fim de favorecer seu bem-estar, sendo assim cabe ao homem proporcionar aos animais um ambiente adequado (BACCARI JR., 2001).

Com base nesse pressuposto, objetivou-se avaliar os parâmetros fisiológicos de bezerros mestiços (Holandês $x$ Gir) no semiárido piauiense.

\section{MATERIAL E MÉTODOS}

O experimento foi realizado no setor de Bovinocultura, do módulo didático produtivo do Campus de Bom Jesus da Universidade Federal do Piauí (UFPI), situado no município de Bom Jesus, sul do estado do Piauí, região do Vale do

AGRARIAN ACADEMY, Centro Científico Conhecer - Goiânia, v.1, n.01; p.103 2014 
Gurgueia (090'28" latitude sul e 44ำ1'31" longitude oeste), a uma altitude de 277 metros.

Foram utilizados 12 bezerros da raça Girolando, sendo seis machos e seis fêmeas, com aproximadamente dois a quatro meses de idade. Esses animais eram mantidos em sistema semi-intensivo, sendo amamentados naturalmente duas vezes ao dia, 05 e 16 horas, soltos no piquete de descanso entre os períodos de amamentação, onde era fornecida alimentação volumosa com capim elefante (Pennisetum purpureum) cv. Roxo, picado.

Os parâmetros fisiológicos, frequência cardíaca (FC), frequência respiratória (FR) e temperatura retal (TR), foram aferidos nessa ordem com os animais em repouso nos períodos da manhã e da tarde, de 06-07 h e 13-14 h, respectivamente, a cada cinco dias, totalizando 10 coletas.

Sendo a FC mensurada em batimentos por minuto, utilizando-se um estetoscópio posicionado entre o terceiro e quarto espaço intercostal esquerdo, a FR mensurada em movimentos respiratórios por minuto, mediante a observação direta dos movimentos do flanco esquerdo; em seguida registrou-se a TR, por meio de um termômetro clínico introduzido diretamente no reto dos animais até o disparo do sonorizador.

As variáveis ambientais, temperatura ambiente (TA) umidade relativa (UR) foram medidas com o auxílio de termo-higrômetro e temperatura do globo negro mediante a um globo-termômetro (termômetro Iconterm 0 a $100^{\circ} \mathrm{C}$ inserido em um globo negro de $150 \mathrm{~mm}$ de diâmetro), instalados em uma altura de $55 \mathrm{~cm}$ do solo, que corresponde a altura média aproximada dos animais. Para o ITGU foi utilizada a equação proposta por BUFFINGTON et al. (1977), em que ITGU = 0,72 (Tbu + Tgn) + 40,6 (onde: Tbu = Temperatura de bulbo úmido em ${ }^{\circ} \mathrm{C} ;$ Tgn $=$ Temperatura de globo negro em $\left.{ }^{\circ} \mathrm{C}\right)$.

O delineamento estatístico utilizado foi o Inteiramente Casualizado em esquema fatorial 2x2 (dois sexos, machos e fêmeas e dois turnos, manhã e Tarde), com oito repetições, utilizando-se o logiciário estatístico SAS (2003), a comparação entre as medias pelo teste $t$.

\section{RESULTADOS E DISCUSSÃO}

Foi constatada diferença $(P<0,05)$ entre os turnos para todas as variáveis bioclimáticas. Avaliando a temperatura do ar, esta caracterizou-se fora da zona de conforto térmico (ZCT) para bovinos, que estabelecem uma faixa de 16 a $28^{\circ} \mathrm{C}$ para bovinos indianos. Fornecer aos animais um ambiente de criação dentro da ZCT é essencial na produção animal, já que eles têm gasto mínimo de energia para mantença, maximizando o crescimento $e$, consequentemente, o seu potencial produtivo (FURTADO et al., 2012)

TABELA 1. Médias das variáveis bioclimáticas registradas durante o período experimental

\begin{tabular}{lccccc}
\hline \multirow{2}{*}{ Turnos } & \multicolumn{5}{c}{ Variáveis bioclimáticas } \\
\cline { 2 - 6 } & TA $\left({ }^{\circ} \mathrm{C}\right)$ & $\mathrm{BU}\left({ }^{\circ} \mathrm{C}\right)$ & UR $(\%)$ & TGN $\left({ }^{\circ} \mathrm{C}\right)$ & ITGU \\
\hline Manhã & $26,42 \pm 0,88 \mathrm{~b}$ & $23,43 \pm 0,46 \mathrm{~b}$ & $75,50 \pm 7,68 \mathrm{a}$ & $28,36 \pm 1,29 \mathrm{~b}$ & $77,88 \pm 3,2 \mathrm{~b}$ \\
Tarde & $32,50 \pm 1,03 \mathrm{a}$ & $25,93 \pm 0,88 \mathrm{a}$ & $58,56 \pm 5,17 \mathrm{~b}$ & $34,75 \pm 2,11 \mathrm{a}$ & $84,28 \pm 2,8 \mathrm{a}$ \\
\hline
\end{tabular}

${ }^{*}$ Média seguida de mesma letra nas colunas não diferem estatisticamente pelo teste t a $5 \%$ de probabilidade. 
Segundo SILVA et al. (2012) a UR está direta e inversamente proporcional a temperatura ambiente, sendo que, 0 aumento da temperatura promove consequentemente a diminuição da umidade relativa do ar.

Foi verificada uma amplitude térmica de $6,08^{\circ} \mathrm{C}$, com superioridade para $\mathrm{O}$ período da tarde, tal resultado corrobora com trabalho realizado por SILVA et al. (2012) sob as mesmas condições climáticas, tais autores atribuíram essa situação à elevada incidência de radiação solar verificada no turno da tarde na região Vale do Gurguéia.

Analisando o índice de temperatura de globo e umidade (ITGU) percebe-se que os maiores valores encontram-se durante a tarde, podendo-se relatar uma amplitude de $6,4^{\circ} \mathrm{C}$, caracterizando 0 ambiente com situação de emergência havendo a necessidade iminente de provimento de instalações que modifiquem essa situação e promovam um ambiente que proporcione conforto aos animais e consequentemente estes possam expressar seu máximo potencial produtivo.

Em análise dos parâmetros fisiológicos avaliados nos diferentes turnos, percebe-se que houve diferença significativa para FR entre turnos para as bezerras, verificando-se maior acúmulo de calor no período da tarde, provavelmente em consequência do acúmulo de calor devido a uma maior incidência de radiação solar neste período, como pode ser verificado através dos parâmetros bioclimáticos (Tabela 1) registrados nesse período.

TABELA 2. Médias das variáveis fisiológicas dos bezerros (machos e fêmeas) Girolando nos diferentes turnos do dia (manhã e tarde) no sul do Piauí

\begin{tabular}{lcc|cccc}
\hline \multirow{2}{*}{ Turnos } & \multicolumn{5}{c}{ Parâmetros Fisiológicos } \\
\cline { 2 - 7 } & \multicolumn{2}{c}{ FR (mov/min) } & \multicolumn{2}{c}{ FC (bat/Min) } & \multicolumn{2}{c}{ TR (C) } \\
\hline & Machos & Fêmeas & Machos & Fêmeas & Machos & Fêmeas \\
\hline Manhã & $42,2 \pm 5,6^{\mathrm{Aa}}$ & $41,4 \pm 6,2^{\mathrm{Aa}}$ & $100,3 \pm 20,8^{\mathrm{Aa}}$ & $101,8 \pm 1,9^{\mathrm{Aa}}$ & $38,3 \pm 0,2^{\mathrm{Aa}}$ & $38,3 \pm 0,3^{\mathrm{Aa}}$ \\
Tarde & $43,4 \pm 7,2^{\mathrm{Ab}}$ & $51,2 \pm 14,9^{\mathrm{Ba}}$ & $97,8 \pm 19,7^{\mathrm{Aa}}$ & $99,5 \pm 2,6^{\mathrm{Aa}}$ & $38,7 \pm 0,2^{\mathrm{Bb}}$ & $39,8 \pm 0,3^{\mathrm{Ba}}$ \\
\hline *ádiany
\end{tabular}

*Médias seguidas de mesma letra minúscula nas linhas e maiúscula nas colunas não diferem entre si pelo teste t a $5 \%$ de probabilidade.

Analisando os diferentes sexos dentro de turno verificou-se que as fêmeas apresentaram uma maior utilização da frequência respiratória durante o turno da tarde, possivelmente na tentativa de dissipação de calor corporal.

Sabe-se, no entanto, que a criação de bezerras nos sistemas de produção leiteira tem como principal objetivo a reposição dos animais no plantel. Para isso, torna-se necessário que estes tenham desenvolvimento satisfatório, livre do máximo de fatores que possam interferir no seu desenvolvimento.

Com relação a FC percebeu-se uma semelhança $(P>0,05)$ entre os sexos e turnos. De acordo com DETWEILER (1996) valores normais situam-se entre 48 a 80 $\mathrm{bat} / \mathrm{min}$. No entanto, no presente estudo, verificou-se tanto para sexo como para turno, valores acima dos padrões de referência para a espécie. Segundo DETWEILER (1996), A FC é controlada pela interação dos centros cardioinibidor e cardioacelerador na medula oblonga, os quais, por sua vez, estão sob a influência do sistema nervoso central, incluindo o hipotálamo e o sistema límbico. A temperatura ambiental, além de outras variáveis fisiológicas, pode alterar o tônus vagal intensificando a atividade dos centros cardioacelerador e vasoconstritor, elevando, portanto a FC. 
Verificou-se efeito de turno sobre a temperatura retal tanto de machos como de fêmeas, provavelmente devido ao aumento da temperatura do ar ser mais intensa no período da tarde (Tabela 1) aliado a uma maior incidência de raios solares, resultando em maior carga térmica adicional proveniente da radiação solar, com consequente aumento da quantidade de calor interno nesse período.

Quando se observa os valores de temperatura retal das bezerras, percebe-se uma superioridade diferindo $(P<0,05)$ dos bezerros no turno da tarde, com maior intensidade de acionamento dos mecanismos termorreguladores como pode ser visto através do relativo aumento da FR desses animais no referido turno.

Percebe-se ainda que bezerras apresentam temperatura retal acima dos padrões de normalidade de acordo com STOBER (1993) que, cita uma variação entre $38,0^{\circ} \mathrm{C}$ a $39,3^{\circ} \mathrm{C}$ com avaliação em ambientes quentes.

O interesse dentro do sistema de produção de leite é produzir novilhas para reposição, sendo assim, deve-se buscar melhorias das condições de criação dos animais do presente estudo, visto que bovinos de alta expressão genética para produção de leite são mais sensíveis aos efeitos do estresse térmico do que aquelas de menor produção, pois em condições climáticas estressantes têm o seu consumo alimentar reduzido, não sendo suas necessidades nutricionais atendidas, implicando no menor desenvolvimento corporal e queda na produção (SILVA \& SOUSA JÚNIOR, 2013).

Para amenizar os efeitos das condições climáticas sobre essas bezerras de reposição, deve-se utilizar estratégias que ofereçam aos animais condições para expressarem o máximo potencial produtivo de modo a elevar o bem-estar animal, a produtividade do rebanho e, consequentemente, a eficiência do sistema produtivo (SILVA et al., 2013; SILVA \& SOUSA JÚNIOR, 2013).

\section{CONCLUSÕES}

Bezerras são mais susceptíveis às condições ambientais estressantes da região do vale do Gurguéia, utilizando em maior intensidade os mecanismos termorreguladores principalmente no turno da tarde.

\section{REFERÊNCIAS}

BACCARI JR., F. 2001. Manejo Ambiental de Vacas Leiteiras em Clima Quente. UEL, Londrina. 142p.

BUFFINGTON, C.S.; COLLAZO-AROCHO, A.; CANTON, G.H. Black globe humidity index (bghi) as comfort equation for dairy cows. St. Joseph: American Society Agricultural Engineers, 1977, 19p.

DETWEILER, D.K. Regulação cardíaca. In: DUKES, H. H. Fisiologia dos animais domésticos. Rio de Janeiro: Guanabara Koogan, 1996. 856 p.

FURTADO, D.A.; PEIXOTO, A.P.; REGIS, J.E.F.; NASCIMENTO, J.W.B.; ARAUJO, T.G.P.; LISBOA, A.C.C. Termorregulação e desempenho de tourinhos Sindi e Guzerá, no agreste paraibano. Revista Brasileira de Engenharia Agrícola e Ambiental, v.16, n.9, p.1022-1028, 2012. 
KENDALL, P.E.; NIELSEN, P.P.; WEBSTER, J.R.; VERKERK, G.A.; LITTLEJOHN, R.P.; MATTHEWS, L.R. The effects of providing shade to lactating dairy cows in a temperate climate. Livestock Science, v.103, p.148-157, 2006.

PERISSINOTTO M.; MOURA D.J.; CRUZ V.F., SOUZA S.R.L.; LIMA K.A.O.; MENDES A.S. Conforto térmico de bovinos leiteiros confinados em clima subtropical e mediterrâneo pela análise de parâmetros fisiológicos utilizando a teoria dos conjuntos fuzzy. Ciência Rural, V.39, n.3, p.1492-1498, 2009.

SAS (Statistical Analysis System). 2003. User's Guide. Cary, NC, SAS Institute Inc. $129 \mathrm{p}$.

SILVA, I.M.; PANDORFI, H.; ALMEIDA, G.L.P.; GUISELINI, C.; CALDAS, A.M. Análise espacial das condições térmicas do ambiente pré-ordenha de bovinos leiteiros sob regimes de climatização. Revista Brasileira de Engenharia Agrícola e Ambiental, v.16, n. 6, p.903-909, 2012.

SILVA, T.P.D.; OLIVEIRA, R.G.; SOUSA JÚNIOR, S.C.; SANTOS, K.R. Efeito da exposição à radiação solar sobre parâmetros fisiológicos e estimativa do declínio na produção de leite de vacas mestiças (Holandês $X$ Gir) no sul do estado do Piauí. Comunicata Scientiae, v.3, n.4, p.299-305, 2012.

SILVA, T.P.D; SOUSA JÚNIOR, S.C. Produção de leite de vacas submetidas a diferentes períodos de exposição à radiação solar no sul do Piauí. Agrarian, v.6, n.21, p.320-325, 2013.

SILVA, T.P.D; SOUSA JÚNIOR, S.C; SANTOS, K.R; MARQUES, C.A.T; TORREÃO, J.N.C. Características termorreguladoras e ganho de peso de cordeiros Santa Inês no sul do estado do Piauí no período de transição seca/águas. Agrarian, v.6, n.20, p.198-204, 2013.

STOBER, M. Identificação, anamnese, regras básicas da técnica do exame clínico geral, In: Exame clínico dos bovinos. 3. ed. Rio de Janeiro: Guanabara Koogan, 1993. $419 \mathrm{p}$.

STRICKLIN W.R.; KAUTZ-SCANAVY C.C. The role of behavior in cattle production: a review of research. Applied Animal Ethology, v. 11, n. 3, p.359-390, 1984. 